\title{
Potencial antioxidante e antimicrobiano de espécies da família Asteraceae
}

FABRI, R.L.; NOGUEIRA, M.S.; DUTRA, L.B.; BOUZADA, M.L.M.; SCIO, E.*

Departamento de Bioquímica, Instituto de Ciências Biológicas, Universidade Federal de Juiz de Fora, Bairro Martelos, CEP: 36036-900, Juiz de Fora-Brasil *elita.scio@uff.edu.br

\begin{abstract}
RESUMO: Espécies da família Asteraceae são amplamente utilizadas na medicina popular para diversos fins terapêuticos. Neste contexto, este estudo teve por objetivo averiguar a atividade antimicrobiana e antioxidante in vitro de extratos metanólicos de plantas pertencentes a Asteraceae, a maioria comumente utilizada na medicina tradicional. A prospecção química dos extratos também foi realizada. A atividade antimicrobiana foi avaliada pelo método de susceptibilidade em microdiluição em caldo e a atividade antioxidante determinada pelo ensaio com o radical DPPH. $O$ extrato da folha de Baccharis dracunculifolia apresentou significativa atividade antimicrobiana para Pseudomonas aeruginosa, Bacillus cereus e Cryptococcus neoformans ( $\mathrm{CIM}=0,005 ; 0,005$ e $0,039 \mathrm{mg} \mathrm{mL}^{-1}$, respectivamente). Os extratos das folhas de Bidens segetum e Matricaria chamomilla foram seletivos para Shigella sonneie $P$. aeruginosa $\left(\mathrm{CIM}=0,005\right.$ e $0,078 \mathrm{mg} \mathrm{mL}^{-1}$, respectivamente). Já as folhas de Acanthospermun australe e Baccharis trimera apresentaram atividade significativa apenas para Candida albicans ( $\left.\mathrm{CIM}=0,039 \mathrm{mg} \mathrm{mL}^{-1}\right)$ enquanto as folhas de Taraxacum officinale foram ativos contra ambas leveduras com CIM 0,039 $\mathrm{mg} \mathrm{mL}^{-1}$. Em relação à atividade antioxidante, os extratos das folhas de $B$. dracunculifolia, $T$. officinale e das inflorescências de $B$. segetum apresentaram significativa atividade com $\mathrm{Cl}_{50}$ de 5,5 e $4 \mu \mathrm{g} \mathrm{mL}^{-1}$, respectivamente. A prospecção química dos extratos identificou presença de compostos como flavonoides, terpenos e outros que podem ser responsáveis pelas atividades observadas.
\end{abstract}

Palavras-chave: Asteraceae, atividade antioxidante, atividade antimicrobiana, fitoquímica

ABSTRACT: Antioxidant and antimicrobial potential of Asteraceae species. Asteraceae species have been largely used in folk medicine for several therapeutic purposes. Thus, the aim of this work was to investigate the in vitro antimicrobial and antioxidant activities of methanol extracts from plants belonging to the Asteraceae family, most of which are commonly used in traditional medicine. Chemical prospecting of extracts was also performed. The antimicrobial activity was evaluated through the broth microdilution susceptibility method and the antioxidant activity was determined through DPPH assay. Leaf extract of Baccharis dracunculifolia presented a significant antimicrobial activity against Pseudomonas aeruginosa, Bacillus cereus and Cryptococcus neoformans $\left(0.005,0.005\right.$ and $0.039 \mathrm{mg} \mathrm{mL}^{-1} \mathrm{MIC}$, respectively). Leaf extracts of Bidens segetum and Matricaria chamomilla were selective for Shigella sonnei and $P$. aeruginosa ( 0.005 and 0.078 $\mathrm{mg} \mathrm{mL}^{-1} \mathrm{MIC}$, respectively). On the other hand, Acanthospermun australe and Baccharis trimera leaves only showed significant activity against Candida albicans $\left(0.039 \mathrm{mg} \mathrm{mL}^{-1} \mathrm{MIC}\right)$, while Taraxacum officinale leaves were active against both yeasts, with $0.039 \mathrm{mg} \mathrm{mL}^{-1} \mathrm{MIC}$. As regards antioxidant activity, extracts of $B$. dracunculifolia and $T$. officinale leaves and $B$. segetum inflorescences presented significant activity, with 5,5 and $4 \mu \mathrm{g} \mathrm{mL}^{-1} \mathrm{IC}_{50}$, respectively. The chemical prospecting identified the presence of flavonoids, terpenes and other compounds which may be responsible for the observed activities.

Key words: Asteraceae, antioxidant activity, antimicrobial activity, phytochemistry

\section{INTRODUÇÃO}

O uso de plantas medicinais para o tratamento de muitas doenças está associado à medicina popular de diferentes partes do mundo
(Araújo \& Leon, 2001). Diferentes culturas dos mais distintos lugares, desenvolvidos ou não, conhecem e utilizam o potencial terapêutico dos vegetais no

Recebido para publicação em 27/05/2009

Aceito para publicação em 07/12/2010

Rev. Bras. PI. Med., Botucatu, v.13, n.2, p.183-189, 2011. 
tratamento de doenças, práticas estas que acompanham o homem desde a pré-história e que evoluíram com ele ao longo dos anos (Coutinho et al., 2004).

O interesse pela descoberta de extratos vegetais com diferentes atividades biológicas tem crescido muito nos últimos anos. Neste contexto, plantas que apresentam atividade antioxidante são de grande interesse visto que a presença de radicais livres está associada a diversos fatores como mutação do DNA, oxidação de proteínas e peroxidação lipídica que contribuem para o desenvolvimento de câncer, diabetes, aterosclerose, processos inflamatórios e envelhecimento (Finkel \& Holbrook, 2000). Da mesma forma, plantas que apresentam atividade antimicrobiana também são de extrema importância devido ao fato de muitos microrganismos apresentarem resistência, não somente aos antibióticos já pré-estabelecidos, como também aos de última geração, causando sérios problemas de saúde pública (Prates \& Bloch-Júnior, 2001).

A família Asteraceae é conhecida pelas propriedades terapêuticas, cosméticas e aromáticas. Já é relatado na literatura o uso medicinal dessa família como antihelmíntico, antiinflamatório, adstringente, colestérico, antihemorrágico, antimicrobiano, diurético, analgésico e antiespasmódico (Portillo et al., 2001; Iscan et al., 2006; Abad \& Bermejo, 2007; Benedek et al., 2007; Jeon et al., 2008).

Assim, esse estudo teve como objetivo investigar o potencial antioxidante e antimicrobiano de extratos vegetais de dez espécies da famíliaAsteraceae utilizadas na medicina popular. Além disso, foi realizada a prospecção química dos extratos a fim de se identificar as principais classes de constituintes químicos.

\section{MATERIAL E MÉTODO}

\section{Material vegetal}

As folhas das espécies Acanthospermum australe (Loefl.) Kuntze (CESJF 47438), Achillea millefolium L. (CESJF 46087), Baccharis dracunculifolia DC. (CESJF 46084), Baccharis trimera (Less.) DC. (CESJF 46074), Eupatorium laevigatum Lam. (CESJF 46085), Galinsoga ciliata (Raf.) S.F. Blake (CESJF 46162), Matricaria chamomilla L. (CESJF 47435), Taraxacum officinale Weber ex F.H. Wigg (CESJF 19759) e Vernonia condensataBaker (CESJF 46086) e as folhas e inflorescências de Bidens segetum Mart. Ex Colla (CESJF 47437) foram coletadas em Juiz de Fora, Minas Gerais, entre 2005 e 2006. As exsicatas foram depositadas no Herbário Leopoldo Krieger da Universidade Federal de Juiz de Fora.

\section{Preparação dos extratos vegetais}

As partes secas das plantas $(50 \mathrm{~g})$ foram pulverizadas e maceradas com metanol $(3 \times 200 \mathrm{~mL})$ por cinco dias à temperatura ambiente. Após esse período, o solvente foi evaporado por pressão reduzida e os respectivos extratos metanólicos foram obtidos. Todos os extratos foram mantidos sob refrigeração $\left(4^{\circ} \mathrm{C}\right)$ até serem submetidos aos bioensaios e à prospecção química (Braga et al., 2007).

\section{Prospecção química}

Uma alíquota de cada extrato ( $\left.1 \mathrm{mg} \mathrm{mL}^{-1}\right)$ foi submetida aos testes de identificação dos constituintes químicos, de acordo com o protocolo descrito por Matos (1997).

\section{Atividade antioxidante (DPPH)}

A atividade antioxidante foi baseada no método de redução do radical 2,2'-difenil-1picrihidrazilo (DPPH.) (Govidarajan et al., 2003). Para realização desse teste, os extratos foram solubilizados em $\mathrm{MeOH}\left(1,0 \mathrm{mg} \mathrm{mL}^{-1}\right)$ e diluídos em solução metanólica do radical DPPH $\left(50 \mu \mathrm{mol} \mathrm{L}^{-1}\right)$ (Sigma, St. Louis, MO, EUA) nas concentrações de 250 a $1,95 \mu \mathrm{g} \mathrm{mL}^{-1}$. Após um período de 30 minutos, foram realizadas as leituras das absorbâncias das amostras e do branco (MeOH + DPPH) em espectrofotômetro a $515 \mathrm{~nm}$. $\mathrm{A} \mathrm{Cl}_{50}$, que é a concentração da amostra necessária para reduzir em $50 \%$ a concentração inicial do DPPH, foi calculada utilizando o programa estatístico Grafit5. Desta forma, quanto menor o valor de $\mathrm{Cl}_{50}$, maior a atividade antioxidante do extrato. Rutina (Sigma) e $\alpha$-tocoferol (Sigma) foram utilizados como controle positivo. Todos os testes foram realizados em triplicata. Os valores de $\mathrm{Cl}_{50}$ foram expressos como média \pm erro padrão. As diferenças estatísticas foram avaliadas pelo teste ANOVA $(p<0,05)$.

\section{Microrganismos testados}

Os microrganismos testados foram Staphylococcus aureus (ATCC 6538), Pseudomonas aeruginosa (ATCC 15442), Salmonella enterica sorovar typhimurium (ATCC 13311), Shigella sonnei (ATCC 11060), Klebsiella pneumoniae (ATCC 13866), Escherichia coli (ATCC 10536), Bacillus cereus (ATCC 11778), Candida albicans (ATCC 18804) e Cryptococcus neoformans (ATCC 32608).

\section{Determinação da concentração inibitória mínima (CIM) \\ O ensaio de susceptibilidade em} microdiluição em caldo foi realizado usando o método descrito pela NCCLS (2002) para determinação do CIM. As cepas de bactérias foram cultivadas "overnight" a $37^{\circ} \mathrm{C}$ por $24 \mathrm{~h}$ em ágar Mueller-Hinton (Isofar, Duque de Caxias, RJ, Brasil) e as cepas de fungos a $35^{\circ} \mathrm{C}$ por $48 \mathrm{~h}$ em ágar Sabouraud Dextrose (Isofar). Os testes foram realizados utilizando caldo Mueller-Hinton (MHB) (Isofar) para bactérias e RPMI-

Rev. Bras. PI. Med., Botucatu, v.13, n.2, p.183-189, 2011. 
1640 (Sigma) para fungos. Diluições sucessivas de 5,0 a $0,0025 \mathrm{mg} \mathrm{mL}^{-1}$ dos extratos foram preparadas em microplacas de 96 poços. Para isso foram utilizadas soluções estoque de $25 \mathrm{mg} \mathrm{mL}^{-1} \mathrm{em}$ DMSO $1 \%$. Foram transferidos $80 \mu \mathrm{L}$ dessa solução para as microplacas, que já continham $100 \mu \mathrm{L}$ de meio de cultura. Para completar o volume final de $200 \mu \mathrm{L}$, foram adicionados $20 \mu \mathrm{L}$ de inóculo ( $10^{8}$ unidades formadoras de colônias (UFC mL-1), de acordo com a escala turbidimétrica padrão de McFarland). As placas foram incubadas a $37^{\circ} \mathrm{C}$ por $24 \mathrm{~h}$ para bactérias e a $35 \div \mathrm{C}$ por $48 \mathrm{~h}$ para fungos. Os mesmos testes foram realizados simultaneamente com o controle positivo (MHB ou RPMI-1640 + microrganismo) e o controle negativo (MHB ou RPMI-1640 + extrato). Cloranfenicol (Feniclor $\left.{ }^{\circledR}\right)\left(0,500\right.$ a $\left.0,0002 \mathrm{mg} \mathrm{mL}^{-1}\right)$ e anfotericina B (Sigma) $\left(0,010\right.$ a 0,0002 $\left.\mathrm{mg} \mathrm{mL}^{-1}\right)$ foram usados como compostos de referência para bactérias e fungos, respectivamente. A CIM foi calculada como a menor diluição que apresentou completa inibição do crescimento do microrganismo testado que é visualizada pela ausência de turbidez nos poços. Todos os testes foram realizados em duplicatas.

\section{Avaliação quantitativa da atividade antimicrobiana}

De acordo com Eloff (2004) a atividade antimicrobiana total, que indica quantas vezes os compostos biologicamente ativos, presentes em $1 \mathrm{~g}$ de planta, podem ser diluídos e ainda inibir o crescimento de microrganismos, foi calculada como a razão entre a quantidade de material extraído (em $\mathrm{mg}$ ) por $1 \mathrm{~g}$ de planta e a CIM.
Além da atividade total, o percentual de atividade e o índice de susceptibilidade microbiano (ISM) foram calculados de acordo com Bonjar (2004). O percentual de atividade demonstrou o potencial total antimicrobiano dos extratos. Este número representa a quantidade de cepas susceptíveis a um determinado extrato e foi calculado como a razão entre número de cepas susceptíveis aos extratos vegetais e o total de cepas microbianas testadas. O valor encontrado foi multiplicado por cem. Já o ISM foi usado para comparar a susceptibilidade relativa entre cepas microbianas e foi calculado como a razão entre o número de extratos efetivos contra cada cepa microbiana e o total de extratos vegetais testados. O valor encontrado foi multiplicado por cem. O valor de ISM varia de '0' (resistente a todos os extratos) até ' 100 ' (susceptíveis a todos os extratos).

\section{RESULTADO E DISCUSSÃO}

A família Asteraceae possui várias espécies utilizadas na medicina popular. Para verificar o potencial terapêutico de algumas dessas espécies, suas atividades antioxidante e antimicrobiana foram avaliadas.

Os nomes populares, as partes usadas, as principais classes de fitoconstituintes e o rendimento $(\% \mathrm{p} / \mathrm{p})$ dos extratos estão apresentados na Tabela 1. A maioria das espécies apresentou em sua composição compostos fenólicos como flavonoides $(82 \%)$ e taninos $(82 \%)$. Além desses, foram encontrados alcaloides (64\%), triterpenos (36\%), esteroides (64\%), cumarinas (45\%) e antraquinonas $(27 \%)$. As saponinas foram identificadas somente no extrato das folhas de $B$. dracunculifolia e $A$. australe.

TABELA 1. Nome popular, parte usada, rendimento $(p / p)$ e prospecção química dos extratos da família Asteraceae.

\begin{tabular}{|c|c|c|c|c|c|c|c|c|c|c|c|c|}
\hline \multirow[t]{2}{*}{ Nome científico } & \multirow[t]{2}{*}{ Nome popular } & \multirow{2}{*}{$\begin{array}{c}\text { Partes } \\
\text { usadas }^{\text {a }}\end{array}$} & \multirow{2}{*}{$\begin{array}{l}\text { Rendimento } \\
(p / p)\end{array}$} & \multicolumn{9}{|c|}{ Classes de constituintes químicos ${ }^{b}$} \\
\hline & & & & $\mathrm{Al}$ & $\operatorname{Tr}$ & Es & $\mathrm{Sa}$ & $\mathrm{Cu}$ & $\mathrm{Fe}$ & $\mathrm{Ta}$ & $\mathrm{FI}$ & An \\
\hline Acanthospermum australe & Carrapicho rasteiro & $\mathrm{F}$ & $11 \%$ & + & - & + & + & + & + & + & + & - \\
\hline Achillea millefolium & Mil folhas & $\mathrm{F}$ & $9 \%$ & + & - & + & - & - & + & - & + & - \\
\hline Baccharis dracunculifolia & Alecrim-do-mato & $\mathrm{F}$ & $11 \%$ & - & + & - & + & + & + & + & + & - \\
\hline Baccharis trimera & Carqueja & $\mathrm{F}$ & $8 \%$ & - & - & + & - & + & + & + & - & + \\
\hline Bidens segetum & Picão do mato & $\mathrm{F}$ & $10 \%$ & - & - & + & - & - & + & + & + & + \\
\hline Bidens segetum & Picão do mato & I & $8 \%$ & + & + & - & - & - & + & + & + & + \\
\hline Eupatorium laevigatum & Camara & $\mathrm{F}$ & $13 \%$ & + & - & + & - & - & + & + & + & - \\
\hline Galinsoga ciliata & Picão-branco & $\mathrm{F}$ & $12 \%$ & + & + & - & - & - & + & + & + & - \\
\hline Matricaria chamomilla & Camomila & $\mathrm{F}$ & $8 \%$ & - & + & - & - & - & + & + & + & - \\
\hline Vernonia condensata & Boldo-da-Bahia & $\mathrm{F}$ & $18 \%$ & + & - & + & - & + & + & + & - & - \\
\hline Taraxacum officinale & Dente-de-leão & $\mathrm{F}$ & $7 \%$ & + & - & + & - & + & + & - & + & - \\
\hline
\end{tabular}

a Partes usadas: F: folhas; I: inflorescências. ${ }^{b}$ Classes de constituintes químicos: Al - Alcaloides; Tr - Triterpenoides; Es - Esteroides; Sa - Saponinas; Cu - Cumarinas; Fe - Fenois; Ta - Taninos; FI - Flavonoides; An - Antraquinonas. 
A atividade antioxidante foi determinada pelo ensaio com o radical DPPH e os resultados podem ser observados na Tabela 2. O DPPH é um radical cromóforo extremamente estável que apresenta pico de absorção no comprimento de onda de $515 \mathrm{~nm}$ em solução metanólica, pois possui coloração violeta intensa (Arnao, 2000). Conforme o DPPH vai sendo reduzido por antioxidante, o elétron se torna emparelhado e a absortividade desaparece (Brand et al., 1995). Os antioxidantes que reduzem o radical DPPH são caracterizados como primários (Yen \& Chen, 1995). Extratos das folhas de $B$. dracunculifolia, T. officinale e das inflorescências de $B$. segetum apresentaram significativa atividade antioxidante com $\mathrm{Cl}_{50}$ de 5,5 e $4 \mu \mathrm{g} \mathrm{mL}^{-1}$, respectivamente. Esses resultados confirmaram a atividade antioxidante previamente relatada para os extratos de $B$. dracunculifolia (Abad \& Bemejo, 2007) e T. officinale (Hudec et al., 2007; Menghini et al., 2010). A atividade antioxidante desses extratos parece estar relacionada com a presença de compostos fenólicos, como flavonoides, presentes na maioria das espécies estudadas (Osawa, 1994). Substâncias fenólicas como ácido cafeico, ácido clorogênico e luteolina já foram identificadas nas inflorescências de $T$. officinale e tiveram as atividades antioxidantes testadas (Hudec et al., 2007), porém esse é o primeiro relato da atividade antioxidante das folhas dessa espécie.

A atividade antimicrobiana expressa como concentração inibitória mínima (CIM) também está apresentada na Tabela 2. Segundo Rios \& Recio (2005), extratos vegetais com CIM inferiores a 0,100 $\mathrm{mg} \mathrm{mL}^{-1}$ são bastante promissores. Assim, o extrato de $A$. australe apresentou significativa atividade para C. albicans (CIM 0,039 $\mathrm{mg} \mathrm{mL}^{-1}$ ) e o extrato de $B$. dracunculifolia para $P$. aeruginosa, $B$. cereus e $C$. neoformans (CIM 0,005; 0,005; 0,039 $\mathrm{mg} \mathrm{mL}^{-1}$, respectivamente). Os resultados apresentados confirmaram a atividade antimicrobiana já relatada para o extrato de B. dracunculifolia (Abad \& Bemejo, 2007) sendo importante ressaltar que para $P$. aeruginosa, a $\mathrm{CIM}$ foi menor que o controle cloranfenicol. Atividade antiibacteriana já foi relatada para o extrato diclorometânico de A. australe contra Bacillus subtilis, Micrococcus luteus, Listeria monocytogenes e $S$. aureus (Vivot et al., 2007). B. trimera também demonstrou atividade significativa para $C$. neoformans (CIM 0,039 $\mathrm{mg} \mathrm{mL}^{-1}$ ) e, ao contrário de estudos realizados por Avancini et al. (2000) e Betoni et al. (2006), não foi observada atividade para S. aureus. O extrato das folhas de $B$. segetum foi ativo contra $S$.

TABELA 2. Atividade antioxidante e a concentração inibitória mínima (CIM) dos extratos da família Asteraceae.

\begin{tabular}{|c|c|c|c|c|c|c|c|c|c|c|c|}
\hline \multirow[t]{2}{*}{ Nome científico } & \multirow[t]{2}{*}{$P U^{a}$} & Atividade & \multicolumn{9}{|c|}{ Atividade antimicrobiana $\left.(\mathrm{CIM} \mathrm{mg} \mathrm{mL})^{-1}\right)^{c}$} \\
\hline & & & $\mathrm{Sa}$ & $\mathrm{Pa}$ & $\mathrm{Bc}$ & Ss & St & Ec & Kp & $\mathrm{Ca}$ & $\mathrm{Cn}$ \\
\hline Acanthospermum australe & $\mathrm{F}$ & $106 \pm 8,0$ & - & - & - & - & - & - & - & 0,039 & 5 \\
\hline Achillea millefolium & $\mathrm{F}$ & $48 \pm 5$ & - & 5 & 1,25 & 5 & - & - & - & 5 & - \\
\hline Baccharis dracunculifolia & $\mathrm{F}$ & $5 \pm 0,5$ & - & 0,005 & 0,005 & 1,25 & & - & - & - & 0,039 \\
\hline Baccharis trimera & $\mathrm{F}$ & $39 \pm 7$ & - & 5 & 2,5 & 2,5 & - & 5 & - & - & 0,039 \\
\hline Bidens segetum & $\mathrm{F}$ & $52 \pm 16$ & 1,25 & 0,156 & 0,156 & 0,005 & 0,156 & 1,25 & 1,25 & 2,5 & 2,5 \\
\hline Bidens segetum & I & $4 \pm 0,6$ & 5 & 2,5 & 2,5 & 5 & 5 & 5 & 5 & 5 & 2,5 \\
\hline Eupatorium laevigatum & $\mathrm{F}$ & $19 \pm 4,0$ & - & 5 & 1,25 & 5 & 5 & - & - & - & - \\
\hline Galinsoga ciliata & $\mathrm{F}$ & $26 \pm 4,0$ & - & 2,5 & 1,25 & - & 5 & - & - & 2,5 & - \\
\hline Matricaria chamomilla & $\mathrm{F}$ & $35 \pm 0,7$ & 0,313 & 0,078 & 1,25 & - & - & - & - & 5 & 5 \\
\hline Vernonia condens ata & $\mathrm{F}$ & $56 \pm 4,0$ & 5 & 5 & 0,625 & & 1,25 & & 2,5 & - & - \\
\hline Taraxacum officinale & $\mathrm{F}$ & $5,0 \pm 2,0$ & 5 & 2,5 & 2,5 & 2,5 & 5 & 1,25 & 0,625 & 0,039 & 0,039 \\
\hline Rutina $^{d}$ & & $2,5 \pm 0,11$ & & & & & & & & & \\
\hline$\alpha$-tocoferol ${ }^{d}$ & & $0,23 \pm 0,15$ & & & & & & & & & \\
\hline Cloranfenicol $^{\mathrm{e}}$ & & & 0,063 & 0,015 & 0,004 & 0,001 & 0,001 & 0,015 & 0,001 & & \\
\hline Anfotericina $\mathrm{B}^{\mathrm{e}}$ & & & & & & & & & & 0,0004 & 0,0008 \\
\hline
\end{tabular}

a Partes usadas: F - folhas; I - inflorescências. ${ }^{b}$ Resultados expressos como a média \pm erro padrão da média $(n=3)$. ${ }^{c}$ Microrganismos testados: Sa, Staphylococcus aureus; Pa, Pseudomonas aeruginosa; Bc, Bacillus cereus; Ss, Shigella sonnei; St, Salmonella enterica sorovar typhimurium; Ec, Escherichia coli; Kp, Klebsiella pneumoniae; Ca, Candida albicans; Cn, Cryptococcus neoformans. ${ }^{{ }^{\prime}}$ Controles positivos para a atividade antioxidante. ${ }^{e}$ Controles positivos para a atividade antimicrobiana. - Sem atividade. 
sonnei (CIM 0,005 $\mathrm{mg} \mathrm{mL}^{-1}$ ), porém o extrato das inflorescências foi inativo. Não foram encontrados registros prévios sobre a atividade antibacteriana e antifúngica dessa espécie, somente relatos sobre atividade antiviral (Silveira et al., 2009). M. chamomilla foi ativa para $P$. aeruginosa (CIM $0,078 \mathrm{mg} \mathrm{mL}^{-1}$ ), porém estudos realizados por Romero et al. (2005) e Simionatto (2004) não detectaram tal atividade para essa bactéria. T. officinale apresentou atividade somente para as leveduras $C$. albicans e $C$. neoformans (CIM 0,039 mg mL-1), não inibindo 0 crescimento de nenhuma cepa bacteriana.

A atividade total $\left(\mathrm{mL} \mathrm{g}^{-1}\right)$, o percentual de atividade (\%) e o índice de susceptibilidade microbiana - ISM (\%) foram calculados para extratos que apresentaram CIM inferiores a $0,100 \mathrm{mg} \mathrm{mL}^{-1}$. Os resultados observados para atividade total (Tabela 3 ) revelaram interessante atividade antimicrobiana para $B$. dracunculifolia visto que os componentes antimicrobianos dessa espécie podem ser diluídos 22.000 vezes e ainda inibir o crescimento de $P$. aeruginosa e $B$. cereus. $O$ extrato das folhas de $B$. segetum também apresentou expressiva atividade total $\left(20.000 \mathrm{~mL} \mathrm{~g}^{-1}\right)$ contra S. sonnei.

O percentual de atividade que avalia 0 potencial antimicrobiano dos extratos vegetais testados mostrou que o extrato de $B$. dracunculifolia foi o mais ativo (33\%), seguido do extrato de $T$. officinale (22\%) (Figura 1).

O ISM que avalia a susceptibilidade de diferentes cepas de bactérias e fungos para os extratos vegetais mostrou que $C$. neoformans foi o microrganismo mais susceptível (27\%), seguido por C. albicans (18\%) e P. aeruginosa (18\%) (Figura 2).

TABELA 3. Atividade total dos extratos da família Asteraceae com CIM inferior a $0,1 \mathrm{mg} \mathrm{mL}^{-1}$.

\begin{tabular}{lclc}
\hline \multicolumn{1}{c}{ Nome científico } & $\begin{array}{r}\text { Parte } \\
\text { usada }\end{array}$ & \multicolumn{1}{c}{ Microrganismos } & $\begin{array}{c}\text { Atividade total } \\
\left(\mathrm{mL} \mathrm{g}^{-1}\right)\end{array}$ \\
\hline Acanthospermum australe & F & Candida albicans & 2.821 \\
Baccharis dracunculifolia & F & $\begin{array}{l}\text { Pseudomonas aeruginosa } \\
\text { Bacillus cereus }\end{array}$ & 22.000 \\
& & & 22.000 \\
Baccharis trimera & & Cryptococcus neoformans & 2.821 \\
Bidens segetum & F & Cryptococcus neoformans & 2.051 \\
Matricaria chamomilla & F & Shigella sonnei & 20.000 \\
Taraxacum officinale & F & Pseudomonas aeruginosa & 1.025 \\
& F & Candida albicans & 1.795 \\
& & Cryptococcus neoformans & 1.795 \\
\hline
\end{tabular}

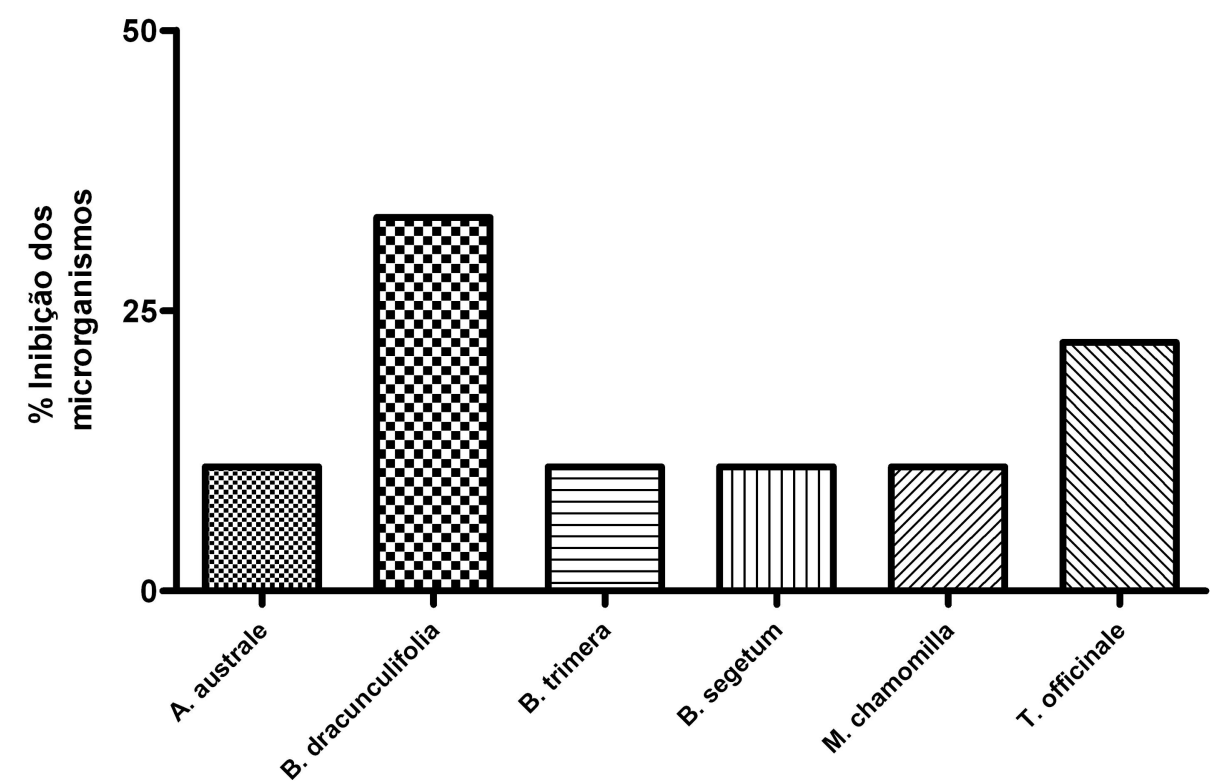

FIGURA 1. Percentual de atividade dos extratos da família Asteraceae com CIM inferiores a $0,1 \mathrm{mg} \mathrm{mL}^{-1}$. 


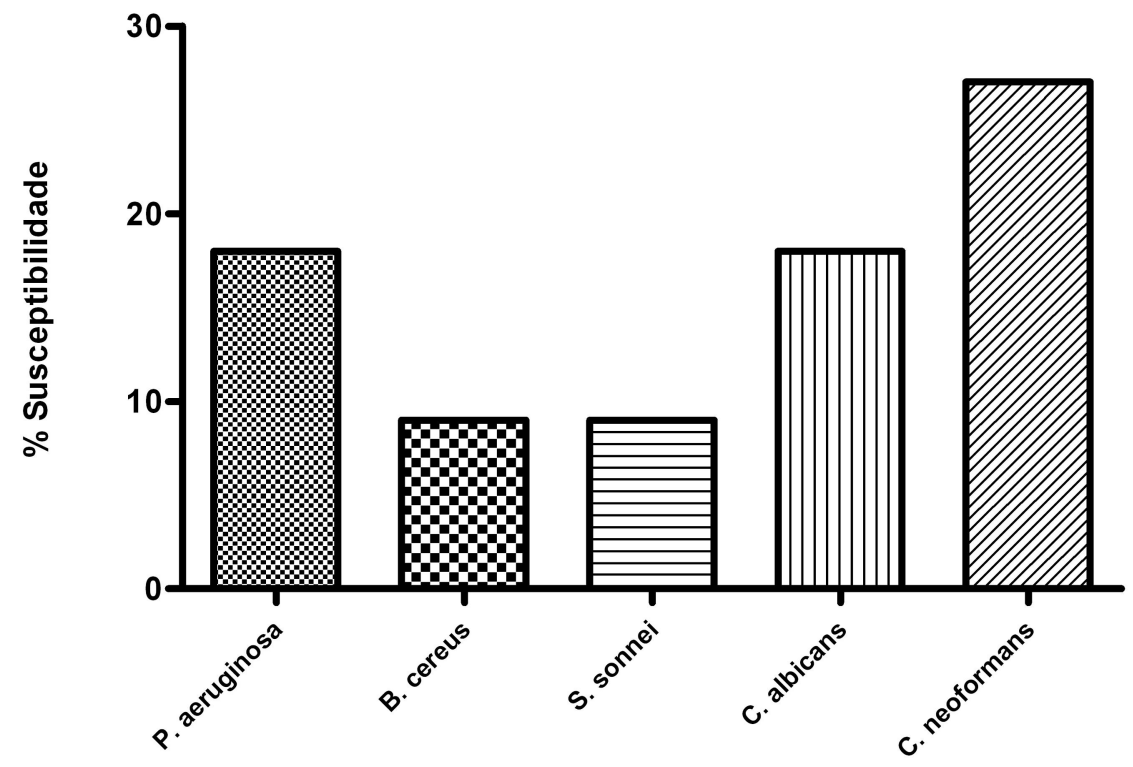

FIGURA 2. Índice de susceptibilidade microbiana (ISM) dos extratos da família Asteraceae com CIM inferiores a $0,1 \mathrm{mg} \mathrm{mL}^{-1}$

\section{CONCLUSÃO}

Neste trabalho foram identificados extratos de espécies da família Asteraceae com significativa atividade antioxidante e antimicrobiana com destaque para as espécies $B$. dracunculifolia e $T$. officinale. Este estudo reafirma a importância de dados etnofarmacológicos na seleção de plantas para triagem de bioatividade uma vez que os resultados apresentaram uma expressiva contribuição para a identificação de atividades biológicas de extratos vegetais da flora brasileira utilizados na medicina popular.

\section{AGRADECIMENTO}

Os autores agradecem a Fundação de Amparo à Pesquisa do estado de Minas Gerais (FAPEMIG) e a Universidade Federal de Juiz de Fora (UFJF) pelo suporte financeiro e à Dra Fátima Regina Salimena pela identificação botânica das espécies vegetais.

\section{REFERÊNCIA}

ABAD, M.J.; BERMEJO, P. Baccharis (Compositae): a review uptade. Arkivoc, v.7, p.76-96, 2007.

ARAÚJO, C.A.C.; LEON, L.L. Biological activities of Curcuma longa L. Memórias do Instituto Oswaldo Cruz, v.96, n.5, p.723-8, 2001.

ARNAO, M.B. Some methodological problems in the determination of antioxidant activity using chromogen radicals: a practical case. Trends in Food Science and Technology, v.11, p.419-21, 2000.

AVANCINI, C.A.M.; WIEST, J.M.; MUNDSTOCK, E.
Bacteriostatic and bactericidal activity of the Baccharis trimera (Less.) DC. - Compositae decocto, as disinfectant or antisseptic. Arquivo Brasileiro de Medicina Veterinária e Zootecnia, v.52, n.3, p.230-4, 2000.

BENEDEK, B.; KOPP, B.; MELZIG, M.F. Achillea millefolium L. s.1.- Is the antiinflamatory activity mediated by protease inhibition? Journal of Ethnopharmacology, v.113, p.3217, 2007.

BETONI, J.E.C.; MANTOVANI, R.P.; BARBOSA, L.N. Synergism between plant extract and antimicrobial drugs used on Staphylococcus aureus diseases. Memórias do Instituto Oswaldo Cruz, v.101, n.4, p.387-90, 2006. BONJAR, G.H.S. New approaches in screening for antibacterials in plants. Asian Journal of Plant Science, v.3, p.55-60, 2004.

BRAND, W.; CUVELIER, M.E.; BERSET, C. Use of a free radical method to evaluate antioxidant activity. Food Science and Technology, v.28, n.1, p.25-30, 1995.

BRAGA, F.G. et al. Antileishmanial and antifungal activity of plants used in traditional medicine in Brazil. Journal of Ethnopharmacology, v.111, n.2, p.396-402, 2007. COUTINHO, M.R. et al. Partial purification of anthocyanins from Brassica oleracea (red cabbage). Separation Science and Technology, v.39, n.16, p.3769-82, 2004. ELOFF, J.N. Quantifying the bioactivity of plant extracts during screening and bioassay-guided fractionation. Phytomedicine, v.11, p.370-1, 2004.

FINKEL, T.; HOLBROOK, N.J. Oxidants, oxidative stress and the biology of ageing. Nature, v.480, p.239-47, 2000. GOVIDARAJAN, R. et al. Studies on the antioxidant activities of Desmodium gangeticum. Biological \& Pharmaceutical Bulletin, v.26, p.1424-7, 2003.

HUDEC, J. et al. Antioxidant capacity changes and phenolics profile of Echinaceae purpurea, Nettle (Urticaria dioica L.), and Dandelion (Taraxacum officinale) after application of polyamine and phenolic biosynthesis regulators. Journal of Agricultural and Food Chemistry, v.55, p.5689-96, 2007. 
ISCAN, G. et al. Biological activity and composition of the essential oil of Achillea schischkinii Sosn. and Achillea aleppica DC. sbsp. aleppica. Journal of Agricultural and Food Chemistry, v.54, p.170-3, 2006.

JEON, H.J. et al. Antiinflamatory activity of Taraxacum officinale. Journal of Ethnopharmacology, v.115, p.82-8, 2008.

MATOS, F.J.A. Introdução à fitoquímica experimental. Fortaleza: Editora EUFC, 1997. 150p.

MENGHINI, L. et al. Antiproliferative, protective and antioxidant effects of artchoke, dandelion, turmeric and rosemary extracts and their formulation. International Journal of Immunopathology and Pharmacology, v.23, n.2, p.601-10, 2010.

NATIONAL COMMITTEE FOR CLINICAL LABORATORY STANDARDS - NCCLS. Reference method for broth dilution antifungal susceptibility testing of yeasts. Approved standard M27-A2 - P. National Committee for Clinical Laboratory Standards. Wayne, PA, 2002.

OSAWA, T. Novel natural antioxidants for utilization in food and biological systems. In: URITANY, I.; GARCIA, V.V.; MENDONZA, E.M. (Eds.). Postharvest biochemistry of plant food-materials in the Tropics. Tokyo: Japan Scientific Society Press, 1994, p.241-51.

PORTILLO, A. et al. Antifungal activity of Paraguayan plants used in traditional medicine. Journal of Ethnopharmacology, v.76, p.93-8, 2001.

PRATES, M.V.; BLOCH-JÚNIOR, C. Peptídeos antimicrobianos. Biotecnology, v.23, p.30-6, 2001.

RIOS, J.L.; RECIO, M. Medicinal plants and antimicrobial plants. Journal of Ethnopharmacology, v.100, p.80-4, 2005. ROMERO, C.D. et al. Antibacterial properties of common herbal remedies of the southwest. Journal of Ethnopharmacology, v.99, n.2, p.253-7, 2005.

SILVEIRA, C.S. et al. In vitro cytotoxic, antioxidant and antiviral effects of Pterocaulon alopecuroides and Bidens segetum extracts. Revista Brasileira de Farmacognosia, v.19, p. 343-8, 2009.

SIMIONATTO, E. Estudos dos constituintes químicos de óleos voláteis de plantas medicinais do Rio Grande do Sul: isolamento, determinação e modificação e atividade biológica. 2004. 232p. Tese de Doutorado (Programa de Pós-graduação em Química) Universidade Federal de Santa Maria, Santa Maria.

VIVOT, E. et al. In vitro antimicrobial activity of six native species from Entre Rios flora (Argentina). Latin American Journal of Pharmacy, v.26, p.563-6, 2007. YEN, G.C.; CHEN, H.Y. Antioxidant activity of various tea extracts in relation to their antimutagenicity. Journal of Agricultural and Food Chemistry, v.43, p.27-32, 1995. 